\title{
T-Inverse Exponential Family Of Distributions
}

\author{
Mahmoud Riad Mahmoud ${ }^{1}, \quad$ Moshera A. M. Ahmad ${ }^{2}$, \\ Azza E. Ismail ${ }^{3, *}$
}

1 Faculty of Graduate Studies for Statistical Research, Cairo University, Giza, Egypt.

2 El Gazeera High Institute for Computer \& Management Information System.

3 Faculty of Graduate Studies for Statistical Research, Cairo University, Egypt.

\begin{abstract}
Recently, several methods have been introduced to generate neoteric distributions with more flexibility, like T-X, T-R $[\mathrm{Y}]$ and alpha power. The T-Inverse exponential $[\mathrm{Y}]$ neoteric family of distributons is proposed in this paper utilising the T-R $[\mathrm{Y}]$ method. A generalised inverse exponential (IE) distribution family has been established. The distribution family is generated using quantile functions of some different distributions. A number of general features in the T-IE [Y] family are examined, like mean deviation, mode, moments, quantile function, and entropies. A special model of the T-IE [Y] distribution family was one of those old distributions. Certain distribution examples are produced by the T-IE [Y] family. An applied case was presented which showed the importance of the neoteric family.
\end{abstract}

Keywords: Inverse exponential distribution, Moments, Mode, quantile function, Shannon's entropy, T-X[Y] family.

\section{Introduction}

[1], indicated that "a distribution is simply a collection of data on a variable". Not all the data follows normal distribution, which is a bellshape curve. The data may be right or left skewed, so what is the best distribution that fits your data? This question is always on researchers' minds. Because the current distributions are not resilient enough to model all the data sets generated by applied fields.

\footnotetext{
${ }^{*}$ coressponding author, E-mail address:azza.e.ismail@gmail.com
} 
Due to the massive improvement in technology, data is growing all the time. It is becoming more complicated as a result of the overlapping of more than one process of generating the data. Therefore, attention is paid to constructing neoteric statistical distributions to deal with the large generated variety of data.

Neoteric distributions families were developed in ad hock manner in the past and [2] showed that most of the approach raised after 1980 was intended to mix two distributions or add an extra parameter to the actual distribution to form a family of neoteric distributions.For example, [3] sugested the skewed normal distribution family by giving an additional parameter to the normal distribution. The exponentiated Weibull distribution was developed by adding another parameter to Weibull distribution by [4]. [5] presented a neoteric framework to create a life distributions family With regard to another's one survival function. [6] explored the family of beta-generated distributions, where he uses beta distribution as a generator function. The cumulative distribution function $\mathrm{CDF}$ of the beta generated distribution is defined as;

$$
G(\nu)=\int_{0}^{F(x)} b(\tau) d \tau,
$$

wherever $F$ is the CDF of any random variable say $X$ and $b(\tau)$ is the PDF of beta distirbution. The beta generated distribution pdf is given by;

$$
g(\nu)=\frac{f(x)}{B(\alpha, \beta)} F^{\alpha-1}(x)(1-F(x))^{\beta-1}, \alpha ; \beta>0,
$$

wherever $\mathrm{B}(\alpha, \beta)$ is the beta function. Several researchers used different $F$ in Equation (1) to generate beta distributions. Instead of beta distribution, Kumaraswamy distribution is applied to expand The beta-generated family of distributions by [7] and [8]. [9] used the first kind of generalized beta-distribution to introduce the generalized Beta-X family. The replacement of a beta PDF with a new PDF for some continous random variable [10] offered a framework to build neoteric distribution families. Their framework implies using $W(F(x))$, that complies with the following requirements:

1. $W(F(x)) \in[c, d]$,

2. $W$ is differentiable and monotonically non-decreasing,

3. $W(F(x)) \rightarrow c$ as $x \rightarrow-\infty$ and $W(F(x)) \rightarrow d$ as $x \rightarrow \infty$,

wherever $[c, d]$ is the support of the random variable $T$ for $-\infty \leq c<d \leq \infty$. [10] named their technique as, the T-X family of distributions. The CDF 
and PDF of T-X family of distributons is supplied as, respectivly;

$$
G(\nu)=\int_{c}^{W(F(x))} r(t) d t=R(W(F(x))),
$$

where $R$ is the CDF of the generated random variable $T$. Furthermore,the corresponding $\mathrm{PDF}$ is

$$
g(\nu)=\left[\frac{d}{d x} W(F(x))\right][r(W(F(x))] .
$$

where $r$ is the PDF of $T$. Several T-X families of distributions were generated using various $W$ functions. The quantile function of a random variable $Y$ was admitted as $W(F(x))$ and the definition of the T-X[Y] family were done by [11]. Several generalized distributions families have released within the literature. T-normal [12], T-gamma [13], T-Cauchy [14], T-Weibull [15], TBurr [16], T-Pareto [17], T-Exponential [18], T-Lomax [19] and T-Dagum [20]. In this paper, we present the T-inverse exponential distribution family, which It applies the T-R[Y] technique explored by [11]. The remaining paper is arranged accordingly. The T-R[Y] technique is briefly reviewed in Section 2. In Section 3, various generic IE subfamilies are listed. Several T-R[Y] distribution general attributes are provided in section 4 . Section 5 some neoteric T-R[Y] distributions. Section 6 introduces some applications.

\section{The Neoteric Distributions Family T-IE[Y]}

The neoteric T-IE[Y] distributions family is introduced in this section, using the T-R[Y] technique described by [11]. This is seen as a generalization of inverse exponential distiribution.

Let us denote the $Y, R$, and $T$ as the random variables with their CDFs $F_{Y}(\nu)=P(Y \leq \nu), F_{R}(\nu)=P(R \leq \nu)$ and $F_{T}(\nu)=P(T \leq \nu)$, respectively. Also, suppose $Q_{Y}(u), Q_{R}(u)$ and $Q_{T}(u)$, the quantile functions coresponding to $Y, R$ and $T$, respectively. Where the definition of the quantile function is $Q_{Z}(u)=\inf \left[z: F_{Z}(z) \geq u\right], 0<u<1$. The PDFs of $Y, R$ and $T$ are pointed as $f_{Y}(\nu), f_{R}(\nu)$ and $f_{T}(\nu)$, respectively. We assume that the random variables $T \in(c, d)$ and $Y \in(l, m)$, for $-\infty \leq c<d \leq \infty$ and $-\infty \leq l<m \leq \infty$. [11] (see also [12]) presented the CDF of the T-R[Y] family as follows:

$$
G_{X}(\nu)=\int_{c}^{Q_{Y}\left(F_{R}(\nu)\right)} f_{T}(\tau) d \tau=F_{T}\left(Q_{Y}\left(F_{R}(\nu)\right)\right) .
$$

From Equation 4 the corresponding PDF is given by;

$$
g_{X}(\nu)=f_{T}\left[Q_{Y}\left(F_{R}(\nu)\right)\right] * Q_{Y}^{\prime}\left(F_{R}(\nu)\right) * f_{R}(\nu) .
$$


Alternatively, Equation 5 can be written as;

$$
g_{X}(\nu)=f_{R}(\nu) * \frac{f_{T}\left(Q_{Y}\left(F_{R}(\nu)\right)\right)}{f_{Y}\left(Q_{Y}\left(F_{R}(\nu)\right)\right)} .
$$

Let the variable $R$ follows the inverse exponential distribution. A $\nu$ variable is claimed to possess an inverse exponential distribution if its PDF and $\mathrm{CDF}$ are, respectively, given by; $f(\nu)=\frac{\vartheta}{\nu^{2}} e^{-\frac{\vartheta}{\nu}}$ and $F(\nu)=e^{-\frac{\vartheta}{\nu}}$, $\nu \geq 0, \vartheta \geq 0$. The CDF and PDF of the T-inverse exponential[Y] family can therefore be provided, respectively, as;

$$
G_{X}(\nu)=\int_{a}^{Q_{Y}\left(e^{-\frac{\vartheta}{\nu}}\right)} f_{T}(\tau) d \tau=F_{T}\left[Q_{Y}\left(e^{-\frac{\vartheta}{\nu}}\right)\right]
$$

and

$$
g_{X}(\nu)=\frac{\vartheta}{\nu^{2}} e^{-\frac{\vartheta}{\nu}} \frac{f_{T}\left[Q_{Y}\left(e^{-\frac{\vartheta}{\nu}}\right)\right]}{f_{Y}\left[Q_{Y}\left(e^{-\frac{\vartheta}{\nu}}\right)\right]}
$$

In cases when $T \sim K w(a, b)$ and $Y \sim \operatorname{uniform}(0,1)$, the T-IE[Y] decreases to the Kumaraswamy-inverse exponential(KW-IE) distribution ([21]).

Proposition 1 The subsequent proposition can easily be proved. Allowing $R$ to be an inverse exponential variable, and $T, Y$ be any random variables with CDFs $F_{T}$ and $F_{Y}$ with $Q$ denoting a quantile function. If $X$ is a random variable following the Equation 7 distribution, It is simple to demonstrate that;

$$
\begin{aligned}
& \text { i } X \stackrel{d}{=} Q_{R}\left(F_{Y}(T)\right), \\
& \text { ii } Q_{X}(u)=Q_{R}\left(F_{Y}\left(Q_{T}(u)\right)\right), \\
& \text { iii } E\left(X^{n}\right)=E\left[\left(Q_{R}\left(F_{Y}(T)\right)\right)^{n}\right], \\
& \text { iv If } T \stackrel{d}{=} Y, \text { then } X \stackrel{d}{=} R \text {. Also, if } Y \stackrel{d}{=} R, \text { then } X \stackrel{d}{=} T .
\end{aligned}
$$

Some recognaized distributions quantile functions (in standard form) are given in Table 1 which are able to be applied to come up with T-IE[Y] sub-families in section 3 .

[22] mentioned that it is useless to use more than five parameters distributions. Using non-standard quantile functions may result more than five parameters T-IE[Y] distributions. Consequently, we concentrate on standard quantile functions during this paper. 
Table 1: Various Y distributions quantile functions.

\begin{tabular}{lll}
\hline No. & $\mathbf{Y}$ & Quantiel functions \\
\hline 1 & Uniform & $v$ \\
2 & Weibull & $-\ln (1-v)$ \\
3 & Log-logistic & $v /(1-v)$ \\
4 & Pareto & $1 /(1-v)$ \\
5 & Logistic & $\ln [v /(1-v)]$ \\
6 & Enutreme Value & $\ln [-\ln (1-v)]$ \\
\hline
\end{tabular}

\section{T-IE[Y] Subfamilies.}

In this part, certain T-IE[Y] subfamilies are shown on the basis of the quantile functions from Table 1.

\subsection{T-IE[Uniform] Family}

Employing uniform distribution quantile function in Table 1, the CDF to Equation 6 is demonstrated as;

$$
G_{X}(\nu)=F_{T}\left(e^{-\frac{\vartheta}{\nu}}\right),
$$

and the PDF to equation 8 is;

$$
g_{X}(\nu)=\frac{\vartheta}{\nu^{2}} e^{-\frac{\vartheta}{\nu}} f_{T}\left(e^{-\frac{\vartheta}{\nu}}\right)
$$

\subsection{T-IE[Weibull] Family}

Employing Weibull distribution quantile function in Table 1, the corresponding CDF to Equation 6 can be deifined as ;

$$
G_{X}(\nu)=F_{T}\left(-\ln \left(1-e^{-\frac{\vartheta}{\nu}}\right)\right)
$$

and the PDF to Equation 10 is;

$$
g_{X}(\nu)=\frac{\vartheta}{\nu^{2}} \frac{e^{-\frac{\vartheta}{\nu}}}{\left(1-e^{-\frac{\vartheta}{\nu}}\right)} f_{T}\left(-\ln \left(1-e^{-\frac{\vartheta}{\nu}}\right)\right) .
$$

\subsection{T-IE[Log-Logistic] Family}

Employing log-logistic distribution quantile function from Table 1, the $\mathrm{CDF}$ to Equation 6 is explored as;

$$
G_{X}(\nu)=F_{T}\left(\frac{e^{-\frac{\vartheta}{\nu}}}{1-e^{-\frac{\vartheta}{\nu}}}\right)
$$


and the PDF to equation 12 is;

$$
g_{X}(\nu)=\frac{\vartheta}{\nu^{2}} \frac{e^{-\frac{\vartheta}{\nu}}}{\left(1-e^{-\frac{\vartheta}{\nu}}\right)^{2}} f_{T}\left(\frac{e^{-\frac{\vartheta}{\nu}}}{1-e^{-\frac{\vartheta}{\nu}}}\right) .
$$

\subsection{T-IE[Pareto] Family}

Employing Pareto distribution quantile function in Table 1, the corresponding CDF to Equation 6 is;

$$
G_{X}(\nu)=F_{T}\left(\frac{1}{1-e^{-\frac{\vartheta}{\nu}}}\right)
$$

and the PDF to Equation 14 is;

$$
g_{X}(\nu)=\frac{\vartheta}{\nu^{2}} \frac{e^{-\frac{\vartheta}{\nu}}}{\left(1-e^{-\frac{\vartheta}{\nu}}\right)^{2}} f_{T}\left(\frac{1}{1-e^{-\frac{\vartheta}{\nu}}}\right) .
$$

\subsection{T-IE[Logistic]Family}

Employing logistic distribution quantile function from Table 1, the corresponding CDF to Equation 6 is;

$$
G_{X}(\nu)=F_{T}\left(-\ln \left(\frac{e^{-\frac{\vartheta}{\nu}}}{1-e^{-\frac{\vartheta}{\nu}}}\right)\right),
$$

and the PDF to equation 16 is;

$$
g_{X}(\nu)=\frac{\vartheta}{\nu^{2}} \frac{1}{\left(1-e^{-\frac{\vartheta}{\nu}}\right)} f_{T}\left(-\ln \left(\frac{e^{-\frac{\vartheta}{\nu}}}{1-e^{-\frac{\vartheta}{\nu}}}\right)\right) .
$$

\subsection{T-IE[Extreme Value] Family}

Employing Extreme Value distribution quantile function from Table 1, the corresponding CDF to Equation 6 is;

$$
G_{X}(\nu)=F_{T}\left(\ln \left(-\ln \left(1-e^{-\frac{\vartheta}{\nu}}\right)\right)\right),
$$

and the PDF to Equation 18 is;

$$
g_{X}(\nu)=\frac{\vartheta}{\nu^{2}} \frac{e^{-\frac{\vartheta}{\nu}}}{\left(1-e^{-\frac{\vartheta}{\nu}}\right)} \frac{1}{\ln \left(1-e^{-\frac{\vartheta}{\nu}}\right)} f_{T}\left(\ln \left(-\ln \left(1-e^{-\frac{\vartheta}{\nu}}\right)\right)\right) .
$$




\section{Several Properities of T-IE[Y] Family}

Several general properties (including transformations, quantile functions, mode, Shannon's entropy, moments and mean deviation) will be detected in this section concerning the T-IE [Y] distributions family.

\subsection{Quantile Function and Transformation}

The realationship among the two random variables $X$ and $T$ is presented in the subsequent lemma.

Lemma 1 Using proposition 1(i) the subsequent lemma is simple to verify. Suppose $T$ is a random variable and $f_{T}(\nu)$ is the corresponding PDF, then;

1. $x=\frac{-\vartheta}{\ln \left(1-e^{-T}\right)}$ follows the T-IE(Weibull) family distribution in Equation 10 .

2. $x=\frac{-\vartheta}{\ln \left(\frac{T}{1+T}\right)}$ follows the T-IE(Log- logistic) family distribution in Equation 12.

3. $x=\frac{-\vartheta}{\ln \left(T-\frac{1}{T}\right)}$ follows the T-IE(Pareto) family distribution in Equation 14.

4. $x=\frac{-\vartheta}{\ln \left(\frac{e^{T}}{1+e^{T}}\right)}$ follows the T-IE(logistic) family distribution in Equation 16 .

5. $x=\frac{-\vartheta}{\ln \left(1-\exp \left(e^{-T}\right)\right)}$ follows the T-IE(Extreme Value) family distribution in Equation 18.

In simple words, the quantile function of any random variable is the inverse of its distribution function. The quantile functions for the T-IE[Y]family of distributions are presented in the next lemma.

Lemma 2 The result of this lemma follows 1 (ii). The quantile function (s) for(1) T-IE (Weibull), (2) T-Ie(log-logistic), (3) T-IE (Pareto),(4) TIE (logistic) and (5) T-IE (Extreme Value) subfamilies of distributions, are respectively, for $0<u<1$;

1. $Q_{x}(u)=\left[\frac{-\vartheta}{\ln \left(1-e^{-Q_{T}^{(u)}}\right)}\right]$,

2. $Q_{x}(u)=\left[\frac{-\vartheta}{\ln \left(\frac{Q_{T}(u)}{1+Q_{T}(u)}\right)}\right]$, 

3. $Q_{x}(u)=\left[\frac{-\vartheta}{\ln \left(1-\exp \left(e^{Q_{T}^{(u)}}\right)\right)}\right]$,
4. $Q_{x}(u)=\left[\frac{-\vartheta}{\ln \left(1-e^{Q_{T}(u)}\right)}\right]$,
5. $Q_{x}(u)=\left[\frac{-\vartheta}{\ln \left(\frac{Q_{T}(u)-1}{Q_{T}(u)}\right)}\right]$.

\subsection{Mode(s)}

Mode is the most value that appears in a set of data and it is important in many applications, like marketing research, media, medical research and business. So, within the current section, the mode(s)'s formula of the T$\mathrm{IE}[\mathrm{Y}]$ family is shown within the next theorem.

Theorem 1 The mode(s) of T-IE/Y] subfamilies of distributions [(1)T-IE(Weibull), (2) T-IE(log-logistic), (3) T-IE(Pareto), (4) T-IE(logistic) and (5) T-IE(Extreme Value)], are the solutions of the subsequent equations, respectively;

$$
\begin{aligned}
& \text { 1. } x=\frac{f_{T}^{\prime}\left(-\ln \left[1-e^{\frac{\vartheta}{\nu}}\right]\right)}{f_{T}\left(-\ln \left[1-e^{\frac{\vartheta}{\nu}}\right]\right)}\left[2-\frac{\vartheta}{\nu}\left(\frac{e^{-\frac{\vartheta}{\nu}}}{1-e^{-\frac{\vartheta}{\nu}}}+1\right)\right], \\
& \text { 2. } x=\frac{f_{T}^{\prime}\left(\frac{e^{-\frac{\vartheta}{\nu}}}{1-e^{-\frac{\vartheta}{\nu}}}\right)}{f_{T}\left(\frac{e^{-\frac{\vartheta}{\nu}}}{1-e^{-\frac{\vartheta}{\nu}}}\right)}\left[2-\frac{\vartheta}{\nu}\left(2 \frac{e^{-\frac{\vartheta}{\nu}}}{1-e^{-\frac{\vartheta}{\nu}}}+1\right)\right], \\
& \text { 3. } x=\frac{f_{T}^{\prime}\left(\frac{1}{1-e^{-\frac{\vartheta}{\nu}}}\right)}{f_{T}\left(\frac{1}{1-e^{-\frac{\vartheta}{\nu}}}\right)}\left[2-\frac{\vartheta}{\nu}\left(2 \frac{e^{-\frac{\vartheta}{\nu}}}{1-e^{-\frac{\vartheta}{\nu}}}+1\right)\right], \\
& \text { 4. } x=\frac{f_{T}^{\prime}\left(\ln \left[\frac{e^{-\frac{\vartheta}{\nu}}}{1-e^{-\frac{\vartheta}{\nu}}}\right]\right)}{f_{T}\left(\ln \left[\frac{e^{-\frac{\vartheta}{\nu}}}{1-e^{-\frac{\vartheta}{\nu}}}\right]\right)}\left[2-\frac{\vartheta}{x}\left(\frac{e^{-\frac{\vartheta}{\nu}}}{1-e^{-\frac{\vartheta}{\nu}}}\right)\right], \\
& \text { 5. } x=\frac{f_{T}\left(\ln \left(-\ln \left[1-e^{\frac{\vartheta}{\nu}}\right]\right)\right)}{f_{T}\left(\ln \left(-\ln \left[1-e^{\frac{\vartheta}{\nu}}\right]\right)\right)}\left[2-\frac{\vartheta}{\nu}\left(\frac{e^{-\frac{\vartheta}{\nu}}}{1-e^{-\frac{\vartheta}{\nu}}}+1\right)\right] .
\end{aligned}
$$

\subsection{Shannon's Entropy}

The entropy term has a wide use in the social scince as a measure of uncertanty. Thus, the shannon's entropy $\left(\eta_{x}\right)$ of a random variable $X$ will be derived in this section as $\eta_{x}=E(-\ln [f(x)])$. 
Theorem 2 T-IE[Y] family Shannon's entropy is given by;

$$
\eta_{x}=\eta_{T}+E\left(\ln f_{y}(T)\right)-\ln [\vartheta]+2 E(\ln \nu)-\vartheta E\left(\frac{1}{\nu}\right)
$$

$\eta_{T}$ represent the Shannon entropy for random variable $T$.

Proof: from proposition 1 (i) $X \stackrel{d}{=} Q_{R}\left(F_{Y}(T)\right)$ and (iv) If $T \stackrel{d}{=} Y$, furthermore $X \stackrel{d}{=} R$ this implies that $T \stackrel{d}{=} Q_{Y}\left(F_{R}(\nu)\right)$. Therefore, from Equation 5 we've

$$
g_{X}(\nu)=f_{R}(\nu) * \frac{\left.f_{T}(T)\right)}{f_{Y}(T)} .
$$

Which implies

$$
\eta_{x}=\eta_{T}+E\left(\ln f_{Y}(T)\right)-E\left(\ln f_{R}(\nu)\right)
$$

Now, $\ln f_{R}(\nu)=\ln [\vartheta]-2 \ln [\nu]-\frac{\vartheta}{\nu}$, which provides

$$
E\left(-\ln \left[f_{R}(\nu)\right]\right)=-\ln [\vartheta]+2 E(\ln \nu)+\vartheta E\left(\frac{1}{\nu}\right) .
$$

from lemma $1 x=\frac{-\vartheta}{\ln \left(1-e^{-T}\right)}$ follows the distribution of T-IE(Weibull), hence

$$
E\left(-\ln \left[f_{R}(\nu)\right]\right)=-3 \ln [\vartheta]-2 E\left(\ln \left[\ln \left[1-e^{-T}\right]\right]\right)-E\left(\ln \left[1-e^{-T}\right]\right),
$$

also $E\left(\ln f_{Y}(T)\right)=E\left(\ln \left[1-e^{\ln \left[1-e^{-T}\right]}\right]\right)$.

So this is the procedures for having shannon's entropy for T-IE(Weibull) and by the same procedures we can get shannon's entropy formulas for all T-IE subfamilies of distributions.

corollary 1 The Shannon's entropies of T-IE/Y] subfamilies of distributions [ (1) T-IE(Weibull), (2) T-IE(log-logistic), (3) T-IE(Pareto), (4) TIE(logistic) and (5) T-IE(Extreme Value)] are given by, respectively;

$$
\begin{aligned}
& \text { 1. } \eta_{x}=\eta_{T}+E\left(\ln \left[1-e^{\ln \left[1-e^{-T}\right]}\right]\right)-3 \ln [\vartheta]-2 E\left(\ln \left[\ln \left[1-e^{-T}\right]\right]\right) \\
& \quad-E\left(\ln \left[1-e^{-T}\right]\right), \\
& \text { 2. } \eta_{x}=\eta_{T}+2 E\left(\ln \left[1-e^{\ln \left[\frac{T}{1+T}\right]}\right]\right)-3 \ln [\vartheta]-E\left(\ln \left[\frac{T}{1+T}\right]\right) \\
& \quad-2 E\left(\ln \left[\ln \left[\frac{T}{1+T}\right]\right]\right), \\
& \text { 3. } \eta_{x}=\eta_{T}+2 E\left(\ln \left[1-e^{\ln \left[1-\frac{1}{T}\right]}\right]\right)-3 \ln [\vartheta]-2 E\left(\ln \left[\ln \left[1-\frac{1}{T}\right]\right]\right) \\
& \quad-E\left(\ln \left[1-\frac{1}{T}\right]\right),
\end{aligned}
$$




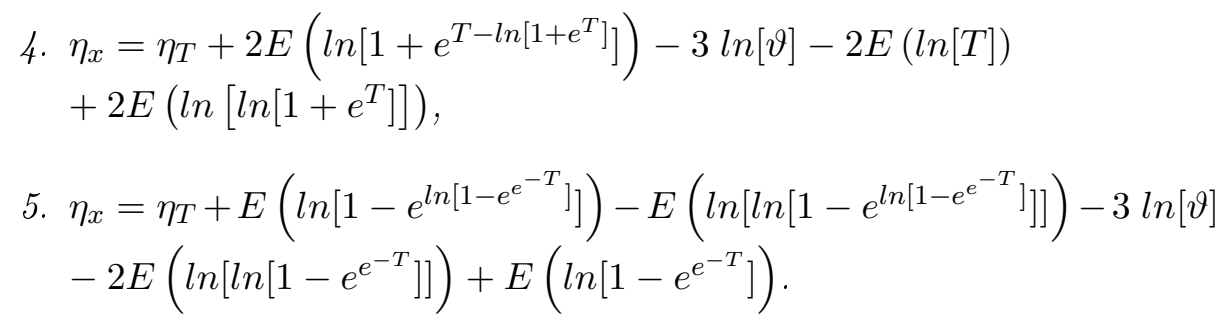

\subsection{Moments}

The usefulness of moments of any statistical distribution is it can describe the shape of any distribution, Thus, in this section, our motivation is to introduce the non-central moments of neoteric T-IE[Y] subfamilies of distributions.

Theorem 3 The relationship among the two random variables $X$ and $T$ is used for developing the non-central moment of the random variable $X$ in lemma 1. The rth non-central moments of T-IE[Y] subfamilies of distributions [ (1) T-IE(Weibull), (2) T-IE(log-logistic), (3) T-IE(Pareto), (4) T-IE(logistic) and (5) T-IE(Extreme Value)] can be respectively defined like that;

$$
\begin{aligned}
& \text { 1. } E\left(x^{r}\right)=\vartheta^{r}-r \sum_{i=0}^{\infty}\left(\begin{array}{c}
i+r \\
i
\end{array}\right) \sum_{j=0}^{i} \frac{(-1)^{i+j}}{-r-j}\left(\begin{array}{l}
i \\
j
\end{array}\right) P_{j, i} E\left[\left(e^{-T}\right)^{-r+i}\right], \\
& \text { 2. } E\left(x^{r}\right)=\vartheta^{r}-r \sum_{i=0}^{\infty}\left(\begin{array}{c}
i+r \\
i
\end{array}\right) \sum_{j=0}^{i} \frac{(-1)^{i+j}}{-r-j}\left(\begin{array}{c}
i \\
j
\end{array}\right) P_{j, i} E\left[\left(\frac{1}{1+T}\right)^{-r+i}\right] \text {, } \\
& \text { 3. } E\left(x^{r}\right)=\vartheta^{r}-r \sum_{i=0}^{\infty}\left(\begin{array}{c}
i+r \\
i
\end{array}\right) \sum_{j=0}^{i} \frac{(-1)^{i+j}}{-r-j}\left(\begin{array}{c}
i \\
j
\end{array}\right) P_{j, i} E\left[\left(\frac{1}{T}\right)^{-r+i}\right], \\
& \text { 4. } E\left(x^{r}\right)=\vartheta^{r}-r \sum_{i=0}^{\infty}\left(\begin{array}{c}
i+r \\
i
\end{array}\right) \sum_{j=0}^{i} \frac{(-1)^{i+j}}{-r-j}\left(\begin{array}{c}
i \\
j
\end{array}\right) P_{j, i} E\left[\left(\frac{1}{1+e^{T}}\right)^{-r+i}\right] \text {, } \\
& \text { 5. } E\left(x^{r}\right)=\vartheta^{r}-r \sum_{i=0}^{\infty}\left(\begin{array}{c}
i+r \\
i
\end{array}\right) \sum_{j=0}^{i} \frac{(-1)^{i+j}}{-r-j}\left(\begin{array}{c}
i \\
j
\end{array}\right) P_{j, i} E\left[\exp \left[e^{-T}\right]\right]
\end{aligned}
$$

Proof: the $r^{\text {th }}$ non-central moment of $X$ can be defined as;

$$
E\left(x^{r}\right)=E\left[\left(Q_{R}\left(F_{Y}(T)\right)\right)^{r}\right] .
$$

For example, the $r^{t h}$ non-central moment of $X$ that follow T-IE [Weibull] distribution can be defined as;

$$
E\left(x^{r}\right)=E\left[\left(\frac{-\vartheta}{\ln \left[1-e^{-T}\right]}\right)^{r}\right]=\vartheta^{r} E\left[\left(-\ln \left[1-e^{-T}\right]\right)^{-r}\right] .
$$


An expansion of the expression $\left(-\ln \left[1-e^{-T}\right]\right)^{-r}$ can be given by using the formula (see [23]);

$$
(-\log [1-z])^{a}=a \sum_{i=0}^{\infty}\left(\begin{array}{c}
i-a \\
i
\end{array}\right) \sum_{j=0}^{i} \frac{(-1)^{i+j}}{a-j}\left(\begin{array}{l}
i \\
j
\end{array}\right) P_{j, i} Z^{a+i}
$$

wherever $a>0$ is any real value and $|z|<1$. The constant $P_{j, i}$ can be calculated using

$$
P_{j, i}=\frac{1}{i} \sum_{m=1}^{i} \frac{(j m-i+m)(-1)^{m}}{m+1} P_{j, i-m}
$$

for $i=1,2,3, \ldots$, and $P_{j, 0}=1$. The rest of non-central moments of T-IE families given in section 3 can be obtained applying the same technique.

\subsection{Mean Deviations}

How far the data values are spread out from the mean value, this is what the mean deviations tells us. $D_{\mu}$ is the mean deviation from the mean.

Theorem $4 D_{\mu}$ for (1) T-IE(Weibull), (2) T-IE(log-logistic), (3) T-IE(Pareto), (4) T-IE(logistic) and (5) T-IE(Extreme Value) subfamilies of distributions, respectively, are;

$$
\begin{aligned}
& \text { 1. } D_{\mu}=2 \mu G(\mu)+2 \vartheta \sum_{i=0}^{\infty}\left(\begin{array}{c}
i+1 \\
i
\end{array}\right) \sum_{j=0}^{i} \frac{(-1)^{-1-j}}{a-j}\left(\begin{array}{c}
i \\
j
\end{array}\right) \\
& P_{j, i} S_{e^{-\frac{\vartheta}{u}}}(\mu, 0,-1+i), \\
& \text { where } S_{\phi(u)}(c, \delta, \alpha)= \\
& \int_{\delta}^{Q_{y}\left(F_{R}(c)\right)}(\phi(u))^{\alpha} f_{T}(u) d u \text { and } Q_{y}\left(F_{R}(c)\right)=-\ln \left[1-F_{R}(c)\right], \\
& \text { 2. } D_{\mu}=2 \mu G(\mu)+2 \vartheta \sum_{i=0}^{\infty}\left(\begin{array}{c}
i+1 \\
i
\end{array}\right) \sum_{j=0}^{i} \frac{(-1)^{-1-j}}{a-j}\left(\begin{array}{l}
i \\
j
\end{array}\right) P_{j, i} \\
& S_{\frac{1}{1+u}}(\mu, 0,-1+i) \text {, where } Q_{y}\left(F_{R}(c)\right)=\frac{F_{R}(c)}{1-F_{R}(c)}, \\
& \text { 3. } D_{\mu}=2 \mu G(\mu)+2 \vartheta \sum_{i=0}^{\infty}\left(\begin{array}{c}
i+1 \\
i
\end{array}\right) \sum_{j=0}^{i} \frac{(-1)^{-1-j}}{a-j}\left(\begin{array}{l}
i \\
j
\end{array}\right) P_{j, i} \\
& S_{\frac{1}{u}}(\mu, 0,-1+i) \text {, where } Q_{y}\left(F_{R}(c)\right)=\frac{1}{1-F_{R}(c)}, \\
& \text { 4. } D_{\mu}=2 \mu G(\mu)+2 \vartheta \sum_{i=0}^{\infty}\left(\begin{array}{c}
i+1 \\
i
\end{array}\right) \sum_{j=0}^{i} \frac{(-1)^{-1-j}}{a-j}\left(\begin{array}{l}
i \\
j
\end{array}\right) P_{j, i} \\
& S_{\frac{1}{1+e^{u}}}(\mu, 0,-1+i) \text {, where } Q_{y}\left(F_{R}(c)\right)=\ln \left[\frac{F_{R}(c)}{1-F_{R}(c)}\right] \text {, }
\end{aligned}
$$



5. $D_{\mu}=2 \mu G(\mu)+2 \vartheta \sum_{i=0}^{\infty}\left(\begin{array}{c}i+1 \\ i\end{array}\right) \sum_{j=0}^{i} \frac{(-1)^{-1-j}}{a-j}\left(\begin{array}{c}i \\ j\end{array}\right) P_{j, i}$
$S_{e^{e^{-T}}}(\mu, 0,-1+i)$, where $Q_{y}\left(F_{R}(c)\right)=\ln \left[-\ln \left[1-F_{R}(c)\right]\right]$.

Proof: The $D_{\mu}$ is defined by

$$
\begin{gathered}
D_{\mu}=E(|\nu-\mu|)= \\
\int_{-\infty}^{\infty}|\nu-\mu| g_{X}(\nu) d \nu=\int_{-\infty}^{\mu}(\mu-\nu) g_{X}(\nu) d \nu+\int_{\mu}^{\infty}(\nu-\mu) g_{X}(\nu) d \nu . \\
D_{\mu}=2 \mu G(\mu)-2 \int_{-\infty}^{\mu} \nu g_{X}(\nu) d \nu .
\end{gathered}
$$

To find the integral in Equation 25, let $I(c)=\int_{-\infty}^{c} \nu g_{X}(\nu) d \nu$. we now present the proof of mean deviation of T-IE(Weibull) family. Using Equation 5 , we rewrite $I(c)$ as;

$$
I(c)=\int_{-\infty}^{c} \nu f_{R}(\nu) \frac{F_{T}\left(Q_{Y}\left(F_{R}(\nu)\right)\right)}{F_{Y}\left(Q_{Y}\left(F_{R}(\nu)\right)\right)} d \nu
$$

Using the substitution $u=Q_{Y}\left(F_{R}(\nu)\right)$ in Equation 26, $I(c)$ can be presented as;

$$
I(c)=\int_{0}^{Q_{Y}\left(F_{R}(\nu)\right)} \vartheta\left(-\ln \left[1-e^{-\frac{\vartheta}{\nu}}\right]\right)^{-1} f_{T}(u) d u .
$$

Using the same technique presented in Equation 22 to write the expansion of $-\ln \left[1-e^{-\frac{\vartheta}{\nu}}\right]$, we get

$$
I(c)=-\vartheta \sum_{i=0}^{\infty}\left(\begin{array}{c}
i+1 \\
i
\end{array}\right) \sum_{j=0}^{i} \frac{(-1)^{-1-j}}{a-j}\left(\begin{array}{l}
i \\
j
\end{array}\right) P_{j, i} S_{e^{-\frac{\vartheta}{u}}}(\mu, 0,-1+i) .
$$

where $P_{i, j}$ is defined in Equation 23 and $S_{\phi(u)}(c, \delta, \alpha)=$ $\int_{\delta}^{Q_{y}\left(F_{R}(c)\right)}(\phi(u))^{\alpha} f_{T}(u) d u$.

\section{A number Of T-IE[Y] Distributions}

Using some different distributions for random variable $\mathrm{T}$ to create several neotric distributions is done in this section. These distributions are, respectivly, Fréchet-IE[Log-logistic], Weibull-IE[Log-logistic] and exponentialIE[Weibull].

\subsection{Fréchet-IE[Log-Logistic] Distribution}

A neoteric three parmeters distribution is introduced in this section, named as Fréchet-IE[log-logistic] distribution, which is an example of T-IE[loglogistic] family of distributions. 


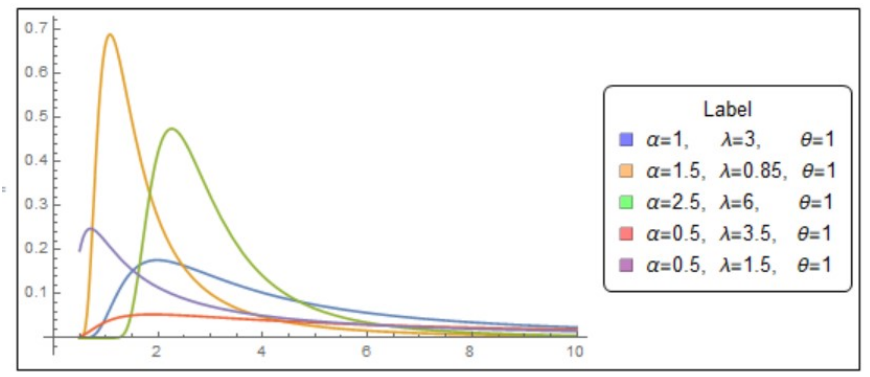

Figure 1: Fréchet-IE[log-logistic] distribution PDFs for different parameters value

If $T$ follow Fréchet distribution, then $f_{T}(\nu)=\alpha \lambda \nu^{-(\alpha+1)} e^{-\lambda \nu^{-\alpha}}$ and $F_{T}(\nu)=e^{-\lambda \nu^{-\alpha}}$. Using Equation 12 and Equation 13, the CDF and PDF of Fréchet-IE[log-logistic] distribution is formed as, respectivly;

$$
\begin{gathered}
G_{X}(\nu)=\exp \left[-\lambda\left(\frac{e^{-\frac{\vartheta}{\nu}}}{1-e^{-\frac{\vartheta}{\nu}}}\right)^{-\alpha}\right], \\
g_{X}(\nu)=\frac{\alpha \lambda \vartheta}{\nu^{2}} \frac{e^{\frac{\alpha \vartheta}{\nu}}}{\left(1-e^{-\frac{\vartheta}{\nu}}\right)^{1-\alpha}} \exp \left[-\lambda\left(\frac{e^{-\frac{\vartheta}{\nu}}}{1-e^{-\frac{\vartheta}{\nu}}}\right)^{-\alpha}\right] .
\end{gathered}
$$

\subsection{Weibull-IE[log-Logistic] Distribution}

This section presents a three-parameter Weibull-IE[log-logistic] distribution. This is a case in point of the T-IE[log-logistic] family. If $T$ follow Weibull distribution, then $f_{T}(\nu)=\frac{\beta}{\eta}\left(\frac{\nu}{\eta}\right)^{\beta-1} e^{-\left(\frac{\nu}{\eta}\right)^{\beta}}$ and $F_{T}(\nu)=1-e^{-\left(\frac{\nu}{\eta}\right)^{\beta}}$. Using Equation 12 and Equation 13, the CDF and PDF of Weibull-IE[loglogistic] distribution is formed as;

$$
\begin{gathered}
G_{X}(\nu)=1-\exp \left[-\frac{1}{\eta}\left(\frac{e^{-\frac{\vartheta}{\nu}}}{1-e^{-\frac{\vartheta}{\nu}}}\right)\right]^{\beta}, \\
g_{X}(\nu)=\frac{\beta \vartheta}{\eta^{\beta} \nu^{2}} \frac{e^{-\frac{\beta \vartheta}{\nu}}}{\left(1-e^{-\frac{\vartheta}{\nu}}\right)^{\beta+1}} \exp \left[-\frac{1}{\eta}\left(\frac{e^{-\frac{\vartheta}{\nu}}}{1-e^{-\frac{\vartheta}{\nu}}}\right)\right]^{\beta} .
\end{gathered}
$$




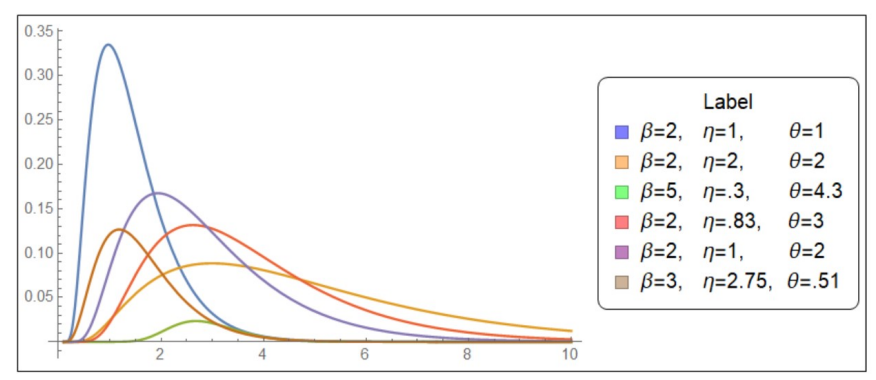

Figure 2: Weibull-IE[log-logistic] distribution PDFs for different parameters value

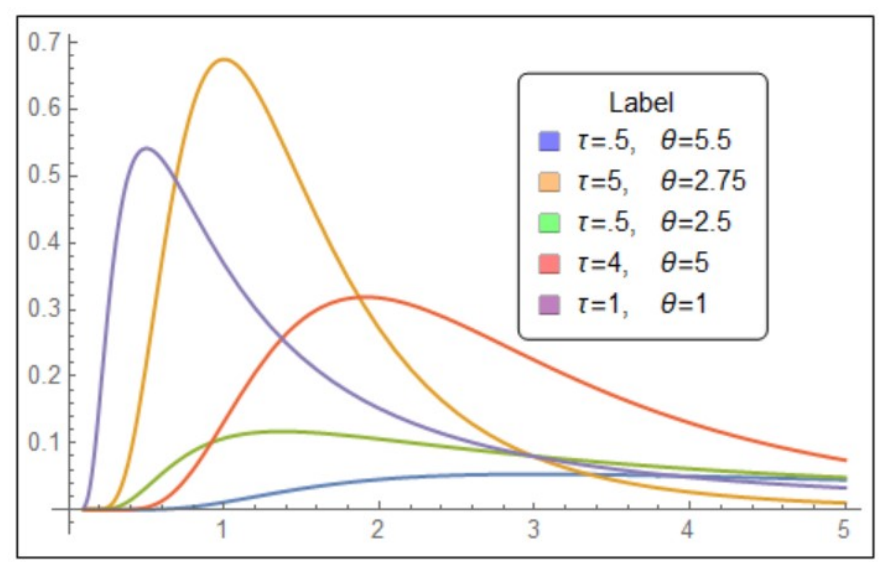

Figure 3: Exp-IE[Weibull] distribution PDFs for different parameters value

\subsection{Exponential-IE[Weibull] Distribution}

This section proposes a two-parameter exponential-IE[Weibull] distribution. This is a case in point of the T-IE[Weibull] family. If $T$ follow exponential distribution, then $f_{T}(\nu)=\gamma e^{-\gamma \nu}$ and $F_{T}(\nu)=1-e^{\gamma \nu}$. Using Equation10 and Equation 11, the CDF and PDF of exponential-IE[Weibull] distribution is formed as;

$$
\begin{gathered}
G_{X}(\nu)=1-\exp \left[-\gamma\left(-\ln \left[1-e^{-\frac{\vartheta}{\nu}}\right]\right)\right] \\
g_{X}(\nu)=\frac{\gamma \vartheta}{\nu^{2}} \frac{e^{\frac{\vartheta}{\nu}}}{1-e^{-\frac{\vartheta}{\nu}}} \exp \left[-\theta\left(-\ln \left[1-e^{-\frac{\vartheta}{x}}\right]\right)\right] .
\end{gathered}
$$

\section{Applications}

The elasticity of Fréchet-IE [log-logistic] is explained by the use of a real data set. A comparison is made between the Fréchet-IE[log-logistic] dis- 
Table 2: AIC, BIC and Log-Likelihood Measures for The Data

\begin{tabular}{llll}
\hline Distribution & AIC & BIC & Log-likelihood \\
\hline Fréchet-IE[Log-Logistic] & 1319.00 & 1327.99 & -656.50 \\
Fréchet & 1557.96 & 1566.95 & -775.97 \\
Inverse power logistic exponential & 1557.96 & 1566.95 & -775.97 \\
Weibull-Lomax[log-logistic] & 5158.78 & 5167.77 & -2576.39 \\
Weibull-exponential & 5160.78 & 5172.77 & -2576.39 \\
Logistic-exponential & 5156.78 & 5162.77 & -2576.39 \\
\hline
\end{tabular}

tribution and various distributions such as (Fréchet, inverse power logistic exponential, Weibull-Lomax[log-logistic], Weibull-exponential and logisticexponential). In monthly renal transplantation patient statistics, we use graft survival times provided in [24].

The log-likelihood value, Bayesian information criterion (BIC) and Akaike information criterion (AIC) are obtained to compare the fitted distributions. The maximum likelihood estimation method is used to estimate distribution parameters. Table 2 contains the values of AIC, BIC and log-likelihood. The figures in Table 2 indicate that the Fréchet-IE[Log-Logistic] distribution is the best-fitted model among the listed model.

\section{References}

[1] T. C. Urdan, Statistics in plain English. Routledge, 2011.

[2] C. Lee, F. Famoye, and A. Y. Alzaatreh, "Methods for generating families of univariate continuous distributions in the recent decades," Wiley Interdisciplinary Reviews: Computational Statistics, vol. 5, no. 3, pp. 219-238, 2013.

[3] A. Azzalini, "A class of distributions which includes the normal ones," Scandinavian journal of statistics, pp. 171-178, 1985.

[4] G. S. Mudholkar and D. K. Srivastava, "Exponentiated weibull family for analyzing bathtub failure-rate data," IEEE transactions on reliability, vol. 42, no. 2, pp. 299-302, 1993.

[5] A. W. Marshall and I. Olkin, "A new method for adding a parameter to a family of distributions with application to the exponential and weibull families," Biometrika, vol. 84, no. 3, pp. 641-652, 1997.

[6] N. Eugene, C. Lee, and F. Famoye, "Beta-normal distribution and its applications," Communications in Statistics-Theory and methods, vol. 31, no. 4, pp. 497-512, 2002. 
[7] M. Jones, "Kumaraswamy's distribution: A beta-type distribution with some tractability advantages," Statistical Methodology, vol. 6, no. 1, pp. 70-81, 2009.

[8] G. M. Cordeiro and M. de Castro, "A new family of generalized distributions," Journal of statistical computation and simulation, vol. 81, no. 7, pp. 883-898, 2011.

[9] C. Alexander, G. M. Cordeiro, E. M. Ortega, and J. M. Sarabia, "Generalized beta-generated distributions," Computational Statistics $\&$ Data Analysis, vol. 56, no. 6, pp. 1880-1897, 2012.

[10] A. Alzaatreh, C. Lee, and F. Famoye, "A new method for generating families of continuous distributions," Metron, vol. 71, no. 1, pp. 63-79, 2013.

[11] M. A. Aljarrah, C. Lee, and F. Famoye, "On generating tx family of distributions using quantile functions," Journal of Statistical Distributions and Applications, vol. 1, no. 1, p. 2, 2014.

[12] A. Alzaatreh, C. Lee, and F. Famoye, "T-normal family of distributions: A new approach to generalize the normal distribution," Journal of Statistical Distributions and Applications, vol. 1, no. 1, p. 16, 2014.

[13] —_, "Family of generalized gamma distributions: Properties and applications," Hacettepe Journal of Mathematics and Statistics, vol. 45, no. 3, pp. 869-886, 2016.

[14] A. Alzaatreh, C. Lee, F. Famoye, and I. Ghosh, "The generalized cauchy family of distributions with applications," Journal of Statistical Distributions and Applications, vol. 3, no. 1, p. 12, 2016.

[15] M. Almheidat, C. Lee, and F. Famoye, "A generalization of the weibull distribution with applications," Journal of Modern Applied Statistical Methods, vol. 15, no. 2, p. 47, 2016.

[16] M. A. Nasir, M. Tahir, F. Jamal, and G. Ozel, "A new generalized burr family of distributions for the lifetime data," Journal of Statistics Applications and Probability, vol. 6, no. 2, pp. 401-417, 2017.

[17] D. Hamed, F. Famoye, and C. Lee, "On families of generalized pareto distributions: properties and applications," Journal of Data Science, vol. 16, no. 2, pp. 377-396, 2018.

[18] M. Zubair, A. Alzaatreh, G. M. Cordeiro, M. Tahir, and M. Mansoor, "On generalized classes of exponential distribution using tx family framework," Filomat, vol. 32, no. 4, pp. 1259-1272, 2018. 
[19] A. A. D. Hamed, "New families of generalized lomax distributions: Properties and applications," International Journal of Statistics and Probability, vol. 8, no. 6, 2019.

[20] M. I. Ekum, M. O. Adamu, and E. E. Akarawak, "T-dagum: A way of generalizing dagum distribution using lomax quantile function," Journal of Probability and Statistics, vol. 2020, 2020.

[21] P. Oguntunde, O. Babatunde, and A. Ogunmola, "Theoretical analysis of the kumaraswamy-inverse exponential distribution," International Journal of Statistics and Applications, vol. 4, no. 2, pp. 113-116, 2014.

[22] N. Johnson, S. Kotz, and N. Balakrishnan, "Equation 14.65 (from continuous univariate distributions, volume 1, )," Continuous Univariate Distributions, vol. 1, 1994.

[23] M. Almheidat, F. Famoye, and C. Lee, "Some generalized families of weibull distribution: Properties and applications," International Journal of Statistics and Probability, vol. 4, no. 3, p. 18, 2015.

[24] A. Algarni, A. M. Almarashi, H. Okasha, and H. K. T. Ng, "E-bayesian estimation of chen distribution based on type-i censoring scheme," Entropy, vol. 22, no. 6, p. 636, 2020. 